\title{
Tracing gas accretion in the Galactic center using isotopic ratios ${ }^{\star}$
}

\author{
D. Riquelme ${ }^{1}$, M. A. Amo-Baladrón ${ }^{2}$, J. Martín-Pintado ${ }^{2}$, R. Mauersberger ${ }^{3}$, S. Martín ${ }^{4}$, and L. Bronfman ${ }^{5}$ \\ ${ }^{1}$ Instituto de Radioastronomía Milimétrica (IRAM), Av. Divina Pastora 7, Local 20, 18012 Granada, Spain \\ e-mail: riquelme@iram.es \\ 2 Centro de Astrobiología (CSIC/INTA), Ctra. de Torrejón a Ajalvir km 4, 28850, Torrejón de Ardoz, Madrid, Spain \\ 3 Joint ALMA Observatory, Av. Alonso de Córdova 3107, Vitacura, Santiago de Chile, Chile \\ ${ }^{4}$ European Southern Observarory, Av. Alonso de Córdova 3107, Vitacura, Casilla 19001, Santiago, Chile \\ 5 Departamento de Astronomía, Universidad de Chile, Casilla 36-D, Santiago, Chile
}

Received 17 May 2010 / Accepted 3 August 2010

\section{ABSTRACT}

\begin{abstract}
Aims. We study the ${ }^{12} \mathrm{C} /{ }^{13} \mathrm{C}$ isotopic ratio in the disk of the central molecular zone and in the halo to trace gas accretion toward the Galactic center region in the Milky Way.

Methods. Using the IRAM 30m telescope, we observed the $J=1-0$ rotational transition of $\mathrm{HCO}^{+}, \mathrm{HCN}, \mathrm{HNC}$, and their ${ }^{13} \mathrm{C}$ isotopic substitutions in order to measure the ${ }^{12} \mathrm{C} /{ }^{13} \mathrm{C}$ isotopic ratio. We observed 9 positions selected throughout the Galactic center region, including clouds at high latitude, locations where the X1 and X2 orbits associated with the barred potential are expected to intersect, and typical Galactic center molecular clouds.

Results. We find a systematically higher ${ }^{12} \mathrm{C} /{ }^{13} \mathrm{C}$ isotopic ratio $(>40)$ toward the halo and the $\mathrm{X} 1$ orbits than for the Galactic center molecular clouds (20-25). Our results point to molecular gas that has undergone a different degree of nuclear processing than observed in the gas towards the inner Galactic center region.

Conclusions. The high isotopic ratios are consistent with the accretion of the gas from the halo and from the outskirts of the Galactic disk.
\end{abstract}

Key words. Galaxy: center - ISM: clouds - ISM: molecules

\section{Introduction}

Many galaxies including our Milky Way contain huge amounts of gas in their central few 100 pc (Mauersberger \& Henkel 1993; Morris \& Serabyn 1996). This gas can be the fuel reservoir that feeds star formation events, and in many cases, the activity of the central object (Usero et al. 2004; García-Burillo et al. 2005). Both processes can occur in bursts. For example, the central region of our Galaxy, which harbors the nearest massive black hole $\left(\sim 4.0 \times 10^{6} M_{\odot}\right.$; Ghez et al. 2005), may have been much more active in the past than it is now (Morris et al. 1999). It is obvious that such a high activity level, even if intermittent, can only be maintained if there is a supply of gas from other regions of the Galaxy, e.g. a disk or halo or from intergalactic space.

Modeling the kinematics of the inner regions of galaxies has been successful in explaining inward motion, matching the observations: Binney et al. (1991) have shown that the large-scale gas kinematics in the Galactic center (GC) can be accounted for by a barred galactic potential. In the dynamical model with a barred potential, there are two major families of stable prograde periodic orbits inside the bar: the $\mathrm{X} 1$ orbits parallel to the bar, and the X2 orbits orthogonal to it (Contopoulos \& Papayannopoulos 1980). In the outer parts of the bar, gas tends to follow the X1 orbits. Farther inwards, these orbits develop cusps and loops (Maciejewski \& Sparke 2000), where matter on self-intersecting orbits gets shocked and falls toward the center, and plunges to orbits of the X2 family (Binney et al. 1991).

\footnotetext{
* Based on observations carried out with the IRAM 30m telescope. IRAM is supported by INSU/CNRS (France), MPG (Germany), and IGN (Spain).
}

Another mechanism for gas accretion toward the central region of galaxies is the disk-halo interaction. A clear example of these interactions has been shown recently by Fukui et al. (2006), who found huge loop structures (known as "giant molecular loops"; GMLs) that connect gas at the Galactic plane with gas at higher latitudes.

However, so far there is no clear observational evidence that gas in the outer disk (in X1 orbits) and/or at high latitudes (in the GMLs) flows toward the central regions. Gas in the GMLs could also be ejected to higher latitudes by winds, originated in past massive star formation events (the GC harbors giant molecular clouds with ongoing massive star formation, e.g. in Sgr B and the 1.3 complex). Observations of gas kinematics tell us what is happening now, but do not allow looking into the past. However, studies of the isotope trace the flow since different regions in a galaxy may have their very characteristic isotopic fingerprints. This approach has the potential to reconstruct the migration history to the central part of the galaxy.

Carbon, nitrogen, and oxygen (CNO) isotopic ratios are diagnostic tools for probing models of Galactic chemical evolution (see e.g. Audouze 1985). In particular, the ${ }^{12} \mathrm{C} /{ }^{13} \mathrm{C}$ isotopic ratio reflects the history of gas proccesing by stars, because this ratio shows the relative degree of primary to secondary processing in stars. While ${ }^{12} \mathrm{C}$ is predicted to be formed in firstgeneration, metal-poor massive stars, on rapid timescales, ${ }^{13} \mathrm{C}$ is thought to be produced primarily via CNO processing of ${ }^{12} \mathrm{C}$ seeds from earlier stellar generations, on a slower timescale in low and intermediate-mass stars or novae (Meyer 1994; Wilson \& Matteucci 1992; Prantzos et al. 1996). The ${ }^{12} \mathrm{C} /{ }^{13} \mathrm{C}$ isotopic ratio is one of the best-established in the GC. It presents a clear 
Table 1. Galactic and equatorial coordinates of the emission-free reference positions.

\begin{tabular}{cccc}
\hline \multicolumn{2}{c}{ Galactic coordinates } & \multicolumn{2}{c}{ Equatorial coordinates } \\
$l\left[^{\circ}\right]$ & $b\left[^{\circ}\right]$ & $\alpha_{J 2000}$ & $\delta_{J 2000}$ \\
\hline 5.75 & 1.0 & $17: 54: 56.6$ & $-23: 29: 13$ \\
4.5 & -1.29 & $18: 00: 57.3$ & $-25: 43: 12$ \\
356.375 & 1.5 & $17: 30: 48.0$ & $-31: 11: 48$ \\
359.75 & -0.25 & $17: 46: 00.1$ & $-29: 16: 47$ \\
1.0 & -1.0 & $17: 51: 52.9$ & $-28: 35: 41$ \\
0.65 & 0.2 & $17: 46: 23.0$ & $-28: 16: 37$ \\
\hline
\end{tabular}

gradient with galactocentric distance (e.g., Wilson 1999), decreasing from 80-90 in the solar neighborhood to 20-25 in the inner Galaxy (toward Sgr A and Sgr B, see e.g., Wannier 1980). However, this ratio is, so far, unknown in the galactic halo and in the X1 orbits.

In this paper we present observations of the $J=1-0$ rotational transition of $\mathrm{HCO}^{+}, \mathrm{H}^{13} \mathrm{CO}^{+}, \mathrm{HCN}, \mathrm{H}^{13} \mathrm{CN}, \mathrm{HNC}$, and $\mathrm{HN}^{13} \mathrm{C}$ to derive the ${ }^{12} \mathrm{C} /{ }^{13} \mathrm{C}$ isotopic ratio toward the gas in the halo, the disk, and for the kinematic components associated with the $\mathrm{X} 1$ and $\mathrm{X} 2$ orbits in the nucleus of our Galaxy. We found very different ${ }^{12} \mathrm{C} /{ }^{13} \mathrm{C}$ isotopic ratios in the $\mathrm{X} 1$ orbits and in the halo from those in the disk and in the X2 orbits. Our findings are consistent with the scenario of less processed gas in the halo and in the $\mathrm{X} 1$ orbits, supporting the idea of gas flowing toward the nucleus of the Milky Way.

\section{Observations}

Observations were carried out with the IRAM-30m telescope at Pico Veleta (Spain) in June and December 2009. We used the E090 band of the new EMIR receiver, which provide a bandwidth of $\sim 8 \mathrm{GHz}$ (from 83.7 to $91.1 \mathrm{GHz}$ ) simultaneously in both polarizations. For the backend, we used the WILMA autocorrelator, providing a resolution of $2 \mathrm{MHz}$ or $6.8 \mathrm{~km} \mathrm{~s}^{-1}$. Observations were performed in position-switching mode where the reference off-positions were checked to be free of emission. Table 1 lists the emission-free positions. The pointing was checked every $2 \mathrm{~h}$ against the source $1757-240$ providing an accuracy better than $5^{\prime \prime}$. Data were calibrated using the standard dual load system. In this work, we use the antenna temperature scale $T_{\mathrm{A}}^{*}$. Main beam temperatures, $T_{\mathrm{MB}}$, can be obtained using $T_{\mathrm{MB}}=F_{\mathrm{eff}} / B_{\mathrm{eff}} \times T_{\mathrm{A}}^{*}$, where the forward efficiency is $F_{\mathrm{eff}}=95 \%$ and the main-beam efficiency is $B_{\text {eff }}=81 \%$ at $86 \mathrm{GHz}$. All positions were observed for no less than 30 min providing an rms noise antenna temperature of about $6 \mathrm{mK}$ in the weakest lines (e.g. $\mathrm{H}^{13} \mathrm{CO}^{+}$).

We observed the nine positions shown in Table 2 displayed on the large-scale map of the $\mathrm{HCO}^{+}$emission shown in Fig. 1. Five of the observed positions are located in the GMLs (halo in Tables 2, 3, and Fig. 1). Three of them are located at the foot points and two at the top of the loops. Two positions are in the disk toward the locations of the expected interactions between the X1 and X2 orbits (Disks X1 and X2 in Tables 2, 3, and Fig. 1). A pair of positions toward the Galactic plane (Disk in Tables 2, 3, and Fig. 1) were used as reference measurements. The positions at the foot points of the GMLs were selected from the intensity peaks of the $\mathrm{SiO}$ maps of Riquelme et al. (2010) and from previous results from higher angular resolution mapping with the Mopra-22 $\mathrm{m}$ telescope (Riquelme et al., in prep.). The Fukui et al. (2006) maps of the GMLs were used to select the position at the top of the loop. The positions in the 1.3 and in
Sgr C complexes correspond to the locations where interactions between the $\mathrm{X} 1$ and $\mathrm{X} 2$ orbits are expected (see, Binney et al. 1991; Stark et al. 2004). The CO and $\mathrm{SiO}(2-1)$ maps of these regions (Tanaka et al. 2007; Amo-Baladrón et al., in prep.) were used for final selection of the positions. The control points in the disk are located in Sgr B2 and in the $l=5.7$ complex selected from Martín et al. (2008) and Fukui et al. (2006), respectively.

\section{Results}

Figure 1 shows the spectra taken in all the observed positions in the $J=1 \rightarrow 0$ transition of the main isotope of $\mathrm{HCO}^{+}, \mathrm{HCN}$ and $\mathrm{HNC}$ and their ${ }^{13} \mathrm{C}$ isotopologues. All the species have been clearly detected in all our sources, except $\mathrm{HN}^{13} \mathrm{C}$, with tentative detections in "Halo 1" and "Halo 3", and a nondetection in "Halo 2".

Table 3 shows the integrated line-intensity ratios between the different isotopologues derived for all positions and velocity components. To distinguish between the different kinematical components, the line intensity ratios were obtained by integrating the line profiles in the velocity ranges given in Table 3. We derived line-intensity ratios from three different species, obtained the highest values from $\mathrm{HCO}^{+}$.

\section{The ${ }^{12} \mathrm{C} /{ }^{13} \mathrm{C}$ isotopic ratios}

The isotopologues that we observed in this work have very similar rotational constants and Einstein coefficients, therefore the beam size is very similar. For optically thin emission, one would expect that line intensity ratios would be directly converted into column density ratios, i.e., $\int T_{\mathrm{H}^{12} \mathrm{CO}^{+}} \mathrm{d} v / \int T_{\mathrm{H}^{13} \mathrm{CO}^{+}} \mathrm{d} v=$ $N\left(\mathrm{H}^{12} \mathrm{CO}^{+}\right) / N\left(\mathrm{H}^{13} \mathrm{CO}^{+}\right)=\left[\mathrm{H}^{12} \mathrm{CO}^{+}\right] /\left[\mathrm{H}^{13} \mathrm{CO}^{+}\right]$.

In general, the molecular isotopologue ratios (see Table 3 ) do not translate directly into ${ }^{12} \mathrm{C} /{ }^{13} \mathrm{C}$ isotopic ratios. Opacity, chemical fractionation, and selective photodissociation effects must be considered to derive the isotopic ratios from molecular lineintensity ratios. In the following, we discuss the importance of these effects on the ${ }^{12} \mathrm{C} /{ }^{13} \mathrm{C}$ isotopic ratios that we derive from our integrated intensity ratio of the isotopologues.

\subsection{Opacity effects, isotopic fractionation, and selective photodissociation}

In our case, it is impossible to estimate the line optical depth from the emission of just one single transition. Since optical depth effects will saturate the emission from the most abundant isotopologue, our derived molecular isotopic ratios must be considered to be lower limits to the actual ${ }^{12} \mathrm{C} /{ }^{13} \mathrm{C}$ isotopic ratios. In almost all cases, the $\mathrm{HCO}^{+} / \mathrm{H}^{13} \mathrm{CO}^{+}$intensity ratios or their limits are higher than those derived from the HCN and HNC isotopomers. This may be interpreted as $\mathrm{HCO}^{+}$being the least optically thick among the three molecular line emissions used in this work. Therefore, $\mathrm{HCO}^{+} / \mathrm{H}^{13} \mathrm{CO}^{+}$intensity ratios give the most stringent limits to the ${ }^{12} \mathrm{C} /{ }^{13} \mathrm{C}$ isotopic ratios. We still cannot exclude that $\mathrm{HCO}^{+}$is also affected by opacity effects, which means that ${ }^{12} \mathrm{C} /{ }^{13} \mathrm{C}$ isotopic ratios are even higher than inferred from the $\mathrm{HCO}^{+} / \mathrm{H}^{13} \mathrm{CO}^{+}$intensity ratios. In the following, we therefore only consider the isotopologue ratios derived from $\mathrm{HCO}^{+}$.

Another important effect may be chemical fractionation (Wilson 1999, and references therein). Langer et al. (1984) studied the fractionation of carbon and oxygen isotopes with a timedependent chemical model. Their model considers a cloud lifetime of $10^{8}$ years, temperatures from 6 to $80 \mathrm{~K}, \mathrm{H}_{2}$ densities 
D. Riquelme et al.: Tracing gas accretion in the Galactic center using isotopic ratios

Table 2. Observed positions.

\begin{tabular}{lccccl}
\hline \hline $\begin{array}{l}\text { Associated } \\
\text { object }\end{array}$ & \multicolumn{2}{c}{ Galactic coordinates } & \multicolumn{2}{c}{ Equatorial coordinates } & This work \\
\hline M+5.3 -0.3 & $l\left[^{\circ}\right]$ & $b\left[^{\circ}\right]$ & $\alpha_{J 2000}$ & $\delta_{J 2000}$ & \\
M-3.8 +0.9 & 5.45 & -0.324 & $17^{\mathrm{h}} 59^{\mathrm{m}} 17.8^{\mathrm{s}}$ & $-24^{\circ} 24^{\prime} 38^{\prime \prime}$ & Halo 1 \\
M-3.8 + 0.9 & 356.206 & 0.83 & $17^{\mathrm{h}} 32^{\mathrm{m}} 59.8^{\mathrm{s}}$ & $-31^{\circ} 42^{\prime} 17^{\prime \prime}$ & Halo 2 \\
Top Loop & 356.179 & 0.925 & $17^{\mathrm{h}} 32^{\mathrm{m}} 33.2^{\mathrm{s}}$ & $-31^{\circ} 40^{\prime} 31^{\prime \prime}$ & Halo 3 \\
Top Loop & 4.75 & -0.8 & $17^{\mathrm{h}} 59^{\mathrm{m}} 34.9^{\mathrm{s}}$ & $-25^{\circ} 15^{\prime} 16^{\prime \prime}$ & Halo 4 \\
1.3 complex & 356.549 & 1.339 & $17^{\mathrm{h}} 31^{\mathrm{m}} 52.5^{\mathrm{s}}$ & $-31^{\circ} 08^{\prime} 19^{\prime \prime}$ & Halo 5 \\
Sgr C & 1.28 & +0.07 & $17^{\mathrm{h}} 48^{\mathrm{m}} 21.9^{\mathrm{s}}$ & $-27^{\circ} 48^{\prime} 19^{\prime \prime}$ & Disk X1-1, Disk X2-1 \\
Galactic plane at $l \sim 55^{\prime} 7$ & 359.446 & -0.124 & $17^{\mathrm{h}} 44^{\mathrm{m}} 46.9^{\mathrm{s}}$ & $-29^{\circ} 28^{\prime} 25^{\prime \prime}$ & Disk X1-2, Disk X2-2 \\
Sgr B2 & 5.75 & 0.25 & $17^{\mathrm{h}} 57^{\mathrm{m}} 46.5^{\mathrm{s}}$ & $-23^{\circ} 51^{\prime} 51^{\prime \prime}$ & Disk 1 \\
\hline
\end{tabular}

Table 3. Intensity ratios from the observed ${ }^{12} \mathrm{C}$ and ${ }^{13} \mathrm{C}$ isotopomers.

\begin{tabular}{lccccc}
\hline \hline Source & $\begin{array}{c}\text { Velocity component } \\
\text { LSR }\left[\mathrm{km} \mathrm{s}^{-1}\right]\end{array}$ & $\begin{array}{c}\text { Velocity range } \\
{\left[\mathrm{km} \mathrm{s}^{-1}\right]}\end{array}$ & $\begin{array}{c}\mathrm{HCO}^{+} / \mathrm{H}^{13} \mathrm{CO}^{+} \\
\text {ratio of } \int T_{\mathrm{A}}^{*} \mathrm{~d} v\end{array}$ & $\begin{array}{c}\mathrm{HCN} / \mathrm{H}^{13} \mathrm{CN} \\
\text { ratio of } \int T_{\mathrm{A}}^{*} \mathrm{~d} v\end{array}$ & $\begin{array}{c}\mathrm{HNC} / \mathrm{HN}^{13} \mathrm{C} \\
\text { ratio of } \int T_{\mathrm{A}}^{*} \mathrm{~d} v\end{array}$ \\
\hline Halo 1 & 100 & {$[50,190]$} & $45.5 \pm 5.4$ & $13.5 \pm 0.2$ & $\geq 25.6$ \\
& 87 & {$[50,97]$} & $\geq 73.9$ & $25.8 \pm 2.0$ & $\geq 7.5$ \\
& 117 & {$[97,135]$} & $32 \pm 3.7$ & $11.3 \pm 0.1$ & $\geq 37.5$ \\
Halo 2 & 144 & {$[135,190]$} & $39.1 \pm 24.7$ & $16.7 \pm 1.8$ & $\geq 2.7$ \\
& -62 & {$[-115,-20]$} & $73.1 \pm 36.5$ & $14.6 \pm 1.0$ & $\geq 15.4$ \\
& left wing & {$[-115,-70]$} & $\geq 34.4$ & $21.2 \pm 3.3$ & $\geq 8$ \\
Halo 3 & right wing & {$[-70,-20]$} & $53.2 \pm 26.1$ & $11.6 \pm 0.9$ & $\geq 13.6$ \\
& -60 & {$[-120,-30]$} & $38 \pm 5.0$ & $13 \pm 0.3$ & $\geq 40.1$ \\
Halo 4 & left wing & {$[-120,-80]$} & $54.2 \pm 37.7$ & $19.2 \pm 1.9$ & $\geq 10.4$ \\
& central peak & {$[-80,-30]$} & $35.7 \pm 4.0$ & $12 \pm 0.2$ & $48.3 \pm 21.8$ \\
& 200 & {$[150,250]$} & $28.3 \pm 5.4$ & $11.8 \pm 0.9$ & $14.9 \pm 3.1$ \\
Halo 5 & peak & {$[150,210]$} & $29.2 \pm 7.5$ & $11.7 \pm 0.8$ & $17.9 \pm 3.9$ \\
Disk X1-1 & right wing & {$[210,250]$} & $\geq 10.6$ & $12.4 \pm 4.0$ & $7.1 \pm 3.4$ \\
& -50 & {$[-100,-40]$} & $13.8 \pm 5.0$ & $6.9 \pm 0.3$ & $22.8 \pm 12$ \\
Disk X2-1 & 180 & {$[140,230]$} & $56 \pm 6.4$ & $10.8 \pm 0.1$ & $25.5 \pm 7.5$ \\
& left wing & {$[140,180]$} & $57.2 \pm 7.5$ & $11.6 \pm 0.1$ & $\geq 8$ \\
& right wing & {$[180,230]$} & $54.4 \pm 11$ & $9.9 \pm 0.2$ & $15.7 \pm 4.4$ \\
Disk X1-2 & 95 & {$[50,140]$} & $29 \pm 1.6$ & $12.1 \pm 0.2$ & $22.1 \pm 2.7$ \\
Disk X2-2 & left wing & {$[50,92]$} & $32.4 \pm 3.7$ & $13.9 \pm 0.4$ & $22.8 \pm 4.0$ \\
Disk 1 & right wing & {$[92,140]$} & $27.3 \pm 1.7$ & $11.3 \pm 0.2$ & $21.7 \pm 8.6$ \\
& 67 & {$[0,100]$} & $42.1 \pm 8.6$ & $9.4 \pm 0.1$ & $9.9 \pm 1.3$ \\
& -43 & {$[-80,-20]$} & $21.7 \pm 1.9$ & $6.6 \pm 0.1$ & $13.1 \pm 1.0$ \\
Disk2 & 66 & {$[35,105]$} & $14.0 \pm 2.8$ & $13.1 \pm 1.0$ & $15.8 \pm 3.6$ \\
& central peak & {$[35,70]$} & $16.4 \pm 4.6$ & $23.1 \pm 3.8$ & $\geq 11.4$ \\
& right wing & {$[70,105]$} & $11.6 \pm 3.4$ & $7.8 \pm 0.7$ & $11.7 \pm 2.7$ \\
& left wing & {$[0,43]$} & $\geq 29.1$ & $9.1 \pm 0.2$ & $\geq 21.9$ \\
& 55 & {$[43,97]$} & $4.1 \pm 0.1$ & $3.5 \pm 0.1$ & $3.7 \pm 0.1$ \\
& right wing & {$[97,135]$} & $16.1 \pm 2.3$ & $13.8 \pm 1.3$ & $\geq 2.6$ \\
\hline & & & & &
\end{tabular}

from $5 \times 10^{2}$ to $1 \times 10^{5} \mathrm{~cm}^{-3}$, and a wide range of metal abundances. Fractionation in $\mathrm{HCO}^{+}$has also been studied by Woods \& Willacy (2009) for protoplanetary disks, which can have a greater impact on the ${ }^{12} \mathrm{C} /{ }^{13} \mathrm{C}$ ratio, but with temperatures and densities different from the GC. Langer et al. (1984) found that the behavior of the carbon isotope ratios can be split into three groups: $\mathrm{CO}, \mathrm{HCO}^{+}$, and "carbon-isotope pool" (which includes the remaining carbon species, such as $\mathrm{C}^{+}, \mathrm{H}_{2} \mathrm{CO}, \mathrm{CS}$, etc). They found that the ${ }^{13} \mathrm{C}$ is enhanced in the $\mathrm{CO}$ (specially at low temperature, low density, and high metal abundance), that the ${ }^{12} \mathrm{C}$ is enhanced in the "carbon isotope pool" group, and that the $\mathrm{HCO}^{+}$ could present both fractionation effects, depending on the physical condition. The behavior of $\mathrm{HCO}^{+}$is related to the formation of this molecule from both $\mathrm{CO}$ and from the "carbon pool isotopes". The formation of $\mathrm{HCO}^{+}$has been explained by the ion-molecule chemistry (Wilson et al. 2009), where the reaction

$\mathrm{H}_{3}^{+}+\mathrm{CO} \leftrightarrow \mathrm{HCO}^{+}+\mathrm{H}_{2}$ likely leads the production of $\mathrm{HCO}^{+} . \mathrm{H}^{13} \mathrm{CO}^{+}$is more tightly bound than $\mathrm{HCO}^{+}$by $0.8 \mathrm{meV}(9 \mathrm{~K})$. The reactions that have the potential to produce isotopic fractionation occurs by protonswitching reactions between formyl ions and carbon monoxide by (Langer et al. 1978, 1984)

$\mathrm{HCO}^{+}+{ }^{13} \mathrm{CO} \leftrightarrow \mathrm{H}^{13} \mathrm{CO}^{+}+{ }^{12} \mathrm{CO}+9 \mathrm{~K}$.

As shown in Fig. 1 of Langer et al. (1984), the isotopic ratio derived from $\mathrm{HCO}^{+}$presents only moderate fractionation. The $\mathrm{H}^{12} \mathrm{CO}^{+} / \mathrm{H}^{13} \mathrm{CO}^{+}$ratio is slightly enhanced at low density and moderate temperature, and decreases at low temperatures and certain densities $\left(n\left(\mathrm{H}_{2}\right)<10^{3}\right.$ and $\left.n\left(\mathrm{H}_{2}\right)>10^{4.5}\right)$. In this model, Langer et al. (1984) considered the formation paths of $\mathrm{HCO}^{+}$ shown in the Table 2 of Graedel et al. (1982). Hüttemeister et al. (1998) and Rodríguez-Fernández et al. (2002) find that the gas in the GC has temperatures ranging from 20 to $200 \mathrm{~K}$. For this temperature range, the predicted fractionation of $\mathrm{HCO}^{+}$is negligible so the fractionation of ${ }^{13} \mathrm{CO}$ cannot occur. In the worst case, the isotopic ratio throughout the GC should not increase 

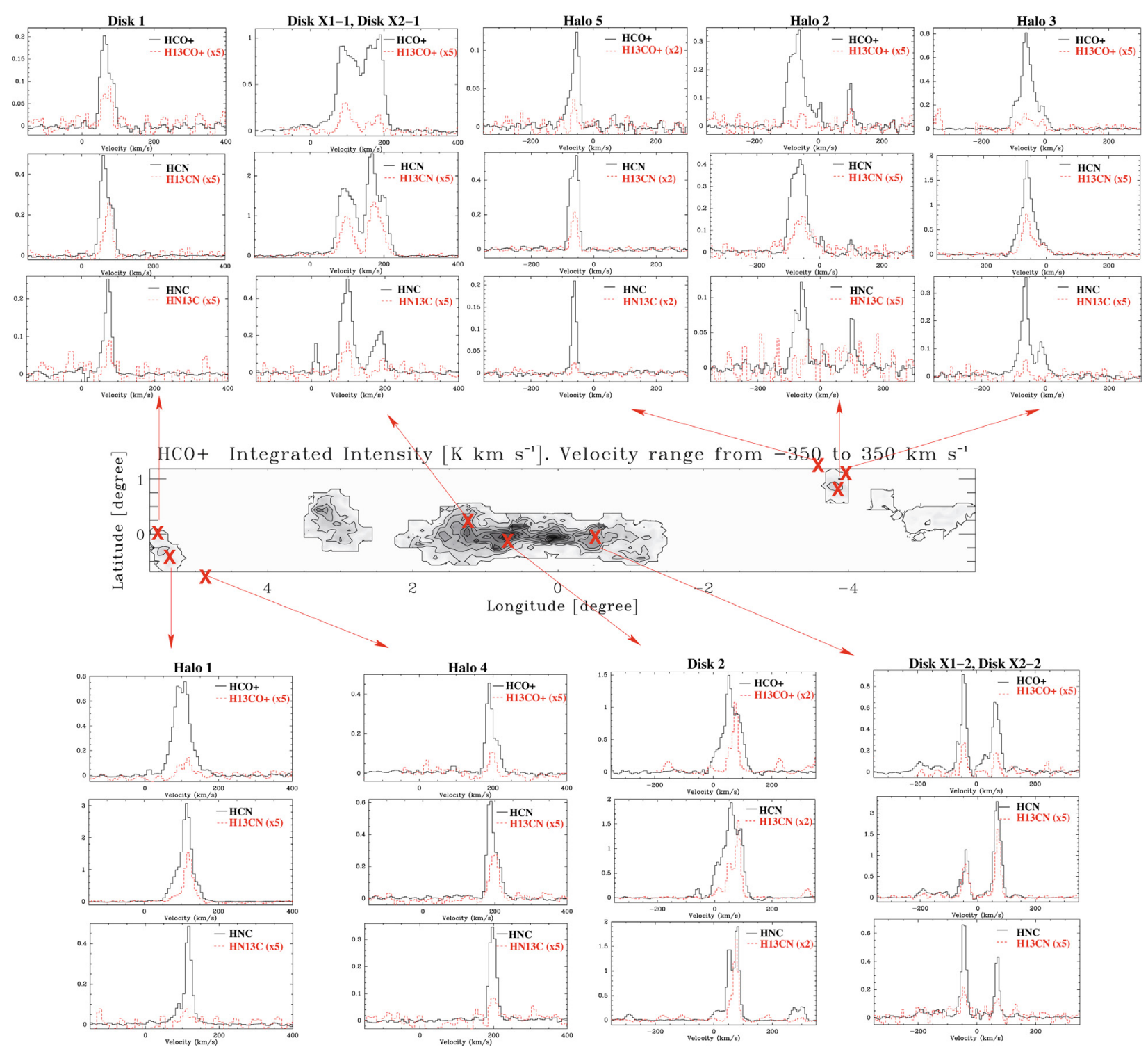

from $-35 x^{\text {to }} 350 \mathrm{~km} \mathrm{~s}^{-1}$
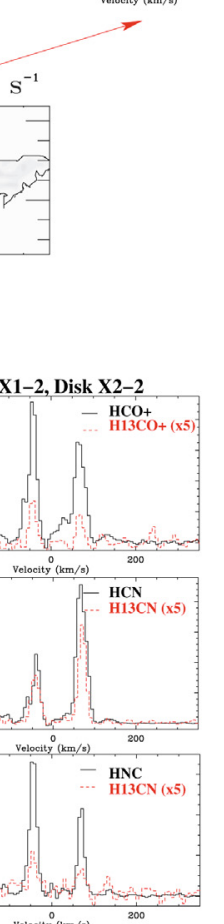

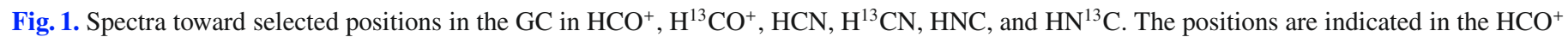
integrated intensity map from Riquelme et al. (2010). The main isotope is plotted in black solid line. The ${ }^{13} \mathrm{C}$ isotope is plotted in red dashed line. The ${ }^{13} \mathrm{C}$ substitution is scaled by a factor of 2 or 5 to allow an easy visualization of the spectra. The factor is indicated in each spectrum.

selectively one of the molecular isotopologue against the other by more than $20 \%$.

For molecular clouds affected by UV radiation, selective photodissociation can take place, which would increase the ${ }^{12} \mathrm{C} /{ }^{13} \mathrm{C}$ isotopic ratio. The more abundant molecules (the main isotopologues) are less affected by photodissociation through self-shielding against UV radiation than the rarer isotopologues. So far, studies of the $\mathrm{HCO}^{+}$and its ${ }^{13} \mathrm{C}$ isotopologue in PDRs have not shown any clear evidence of selective photodissociation (Fuente et al. 2003). Milam et al. (2005) conclude that the ${ }^{12} \mathrm{C} /{ }^{13} \mathrm{C}$ isotopic ratio derived from high-density tracers like $\mathrm{CN}$ are very unlikely to be influenced by isotope-selective photodissociation. However the self-shielding and photodissociation of CO (e.g., Bally \& Langer 1982; Chu \& Watson 1983; Glassgold et al. 1985) could affect our derived ${ }^{12} \mathrm{C} /{ }^{13} \mathrm{C}$ ratios through formation of $\mathrm{HCO}^{+}$via the reaction in Eq. (1). When modeling this effect, Chu \& Watson (1983) concluded that the selective photodissociation will have a negligible impact on the ${ }^{13} \mathrm{CO} /{ }^{12} \mathrm{CO}$ ratio. Although the local enviroment of the GMLs is so far unknown, there are no signposts of UV radiation. There is only a nearby ultracompact H II region identified at $(l, b) \sim$ (356.25, 0.7), but its radial velocity of $\sim 120 \mathrm{~km} \mathrm{~s}^{-1}$ suggests that it is not associated with the foot points of the GMLs (Torii et al. 2010), making it very unlikely that there is any effect on our derived isotopic ratios by photodissociation. The low ${ }^{13} \mathrm{CO} /{ }^{12} \mathrm{CO}$ ratio observed in the GMLs by Torii et al. (2010) does not confirm that the bulk of the gas is neither self-shielded in ${ }^{12} \mathrm{CO}$ nor photodissociated in ${ }^{13} \mathrm{CO}$. The low ratios likely comes from optically thick emission in ${ }^{12} \mathrm{CO}$, suggesting rather high CO column densities. Then the bulk of the gas will not be affected by the rather low fluxes of far UV radiation inferred from the lack of H II regions (Torii et al. 2010) in the area. Under these conditions, it is highly unlikely that the fractionation/selfshielding effect in the ${ }^{13} \mathrm{CO} /{ }^{12} \mathrm{CO}$ could explain the observed high $\mathrm{H}^{12} \mathrm{CO}^{+} / \mathrm{H}^{13} \mathrm{CO}^{+}$ratios. We conclude that the line-intensity ratios derived from our data correspond to a lower limit to the actual ${ }^{12} \mathrm{C} /{ }^{13} \mathrm{C}$ isotopic ratio.

We derived $\mathrm{H}^{12} \mathrm{CO}^{+} / \mathrm{H}^{13} \mathrm{CO}^{+}$intensity ratios from 4 to $>74$. The lowest value of 4 is found toward the source Disk 2 in the position of Sgr B2, where one would expect a value of $\sim 25$. Such a 


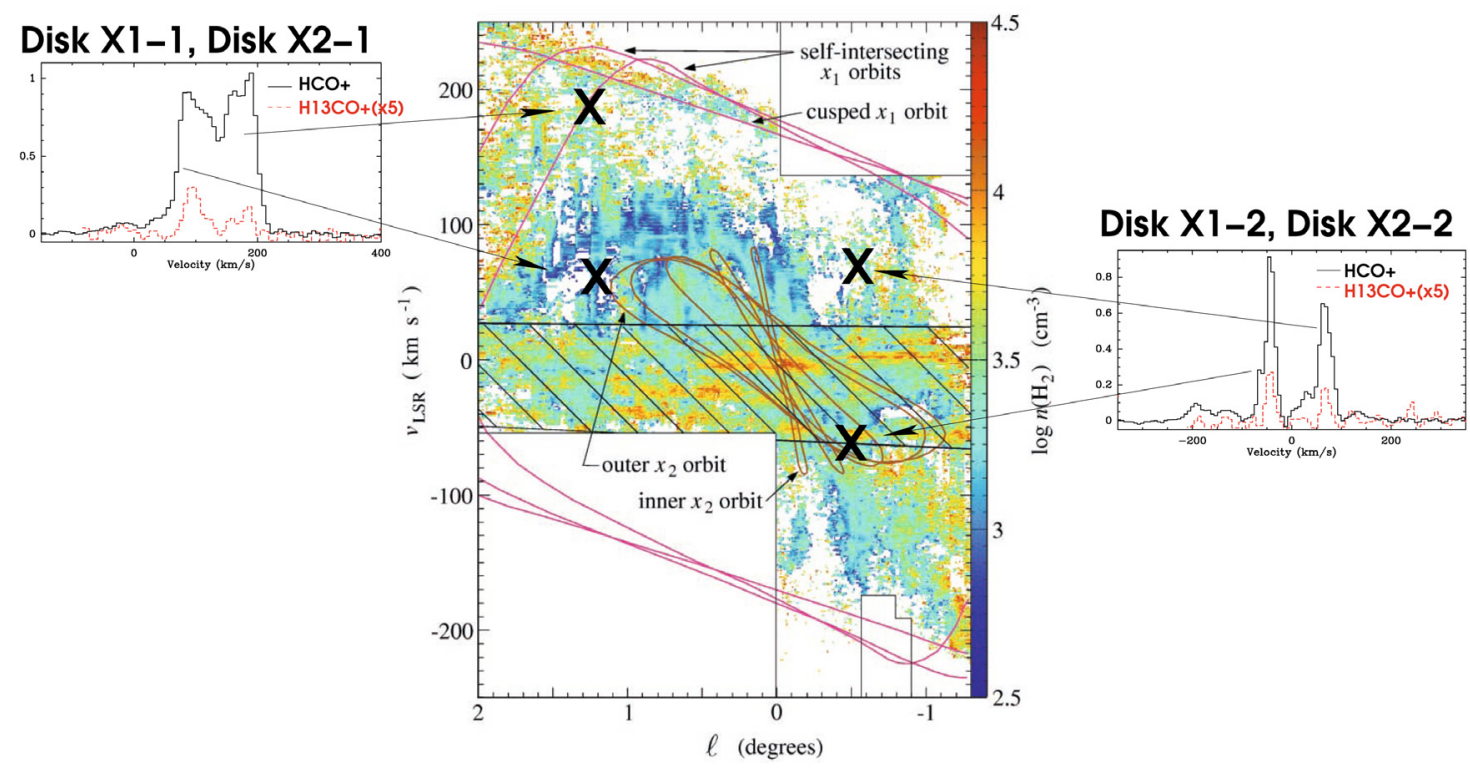

Fig. 2. Spectra toward the $\mathrm{X} 1$ and $\mathrm{X} 2$ orbits in $\mathrm{HCO}^{+}$and $\mathrm{H}^{13} \mathrm{CO}^{+}$(scaled by a factor of 5). Left: spectra toward the 1.3 complex. Center: 1-v diagram showing the $\mathrm{X} 1$ and $\mathrm{X} 2$ orbits superimposed (Stark et al. 2004). Right: spectra toward Sgr C region.

low measured value indicates that $\mathrm{HCO}^{+}$is optically thick. From the results in Table 3, we find a systematic trend in the isotopologue ratios with the highest values, $>74$, found toward Halo 1 and Halo 2. Also Halo 3 and Disk X1-1 and Disk X1-2 show high values of $>42$.

\section{Discussion}

It is remarkable that there is a systematic trend by the ${ }^{12} \mathrm{C} /{ }^{13} \mathrm{C}$ isotopic ratio, found toward the halo and in the disk for the kinematic components associated with the X1 orbits, to be systematically higher by a factor of at least 2 than the standard value in the GC of $\sim 25$. This suggests that there is less processed material in the halo and in the $\mathrm{X} 1$ orbits than in the $\mathrm{X} 2$ orbits and the reference GC positions in the disk.

Surprisingly, the isotopic ratios measured in the Halo 4 and Halo 5 positions at the top of the GMLs show a lower ${ }^{12} \mathrm{C} /{ }^{13} \mathrm{C}$ isotopic ratio than in the other halo positions, which are closer to the "standard" values of the disk. From our data, it is still not clear if this low isotopic ratio is comes from opacity effects in the main isotope or from a mixture with more processed gas from stellar nucleosynthesis in the GC region, likely ejected to high latitudes from the disk.

\subsection{History of the chemical evolution of the molecular gas in the Galactic center}

The lower limits to the ${ }^{12} \mathrm{C} /{ }^{13} \mathrm{C}$ isotopic abundance ratios derived from our $\mathrm{HCO}^{+}$data toward the "typical" GC molecular clouds (Disk 1, Disk 2, Disk X2-1, Disk X2-2) range between 4 to 32, with an average value of 20 . These results agree with the values found in the literature for the GC (20-25, Wilson 1999) and with the average value derived by Riquelme et al. (2010) of 19.8 (from the $\mathrm{HCO}^{+} / \mathrm{H}^{13} \mathrm{CO}^{+}$integrated intensity ratio throughout the GC region), showing a considerable nuclear processing. In contrast, we have found much higher limits to the isotopic ratios toward the locations where gas in the inner disk ${ }^{1}$ is expected to

\footnotetext{
1 Throughout this work, all the positions observed and discussed are in the Galactic center region (in the central kpc of the Galaxy). We denote
}

be interacting with gas in the halo and in the disk. Indeed, the gas in the X1 orbits has a different nature than in the X 2 orbits, as clearly reflected by their different isotopic ratios of $\sim 55$ and 21-30, respectively. Furthermore, in the halo, the interaction occurs when the gas in the top of the loops flows toward the foot points of the GMLs. In both cases, the isotopic ratio reflects a different nature of the gas than in the GC. The ${ }^{12} \mathrm{C} /{ }^{13} \mathrm{C}$ isotopic ratio of the gas in the halo and in the $\mathrm{X} 1$ orbits are in general higher than 40, reaching values over 70 in "Halo 1 ".

Previous studies show a clear gradient in the ${ }^{12} \mathrm{C} /{ }^{13} \mathrm{C}$ ratio with galactocentric distance. The ratio changes from a value of about 50 in the inner Galaxy (4 kpc) to nearly 70 in the local ISM, and $\sim 90$ in the solar system, with a value $\sim 20-25$ in the GC (Wilson 1999), which reflects higher nuclear processing toward the inner Galaxy. Studies of the isotopic ratios in the GC (Wannier 1980; Stark 1981; Henkel et al. 1985; Langer \& Penzias 1990) have shown that the interstellar gas in the GC is in an advanced state of chemical evolution (which corresponds to an enrichment of $\sim 3-4$ with respect to the local gas). This gradient indicates that the material is flowing to the inner central kiloparsec of the GC from the Galactic halo (in the case of GMLs) and the outskirts of the Galactic disk (in the case of X1$\mathrm{X} 2$ interacting orbits).

\subsection{The origin of molecular gas in the GML}

Our lower limits to the ${ }^{12} \mathrm{C} /{ }^{13} \mathrm{C}$ isotopic ratios in the halo sources for the foot points of the GMLs are close to the values measured in the local ISM (69 \pm 6 , Wilson 1999). Based on energetic and morphological arguments, Fukui et al. (2006) argue that it is impossible that the loop features are created by supershells or supernova explosions. Our data strongly support their claim. If the loop features were formed by supernova or hypernova explosions, the gas at the foot points should have been ejected from the $\mathrm{GC}$ to high latitudes, reflecting the isotopic ratio found throughout the GC. It is likely that the gas in the GMLs has been accreted

"disk" as the disk in the CMZ, "inner disk" to the allowed velocities in the X2 orbits, and "outer disk" and "outskirts of the disk" as the noncircular motions (X1 orbits). 
from high latitudes. However, if the low isotopologue ratio found at the top of the loops is confirmed to be the actual isotopic ratios, this might indicate that some gas in the halo could also have been ejected from the disk.

Magneto-hydrodynamical simulations have been successful in explaining the formation of the loops (Matsumoto et al. 1988; Horiuchi et al. 1988; Fukui et al. 2006; Machida et al. 2009; Takahashi et al. 2009). They claim that the GMLs have been formed as a natural consequence of a differentially rotating magnetized gas disk under a strong gravitational potential (Machida et al. 2009). It is found that the loop efficiently accumulates gas generating a dense layer at the top of the loops. However, our results are not consistent with the magneto-hydrodynamical loop scenario in which the gas in the GML falling to their foot point should share the same isotopic ratios as the gas in the Galactic disk. Morris (2006) also point out that there is too much gas left at the top of the loops if the gas is flowing down along the magnetic field lines. Morris (2006) and Torii et al. (2009) alternatively propose that ambient $\mathrm{HI}$ gas may be converted in the GML into $\mathrm{H}_{2}$ during flotation. It is likely that the loops are surrounded by H I gas. The rising portion of the magnetic loops suffer shocks that compress the relatively rarefied atomic gas in front of it, leading to rapid cooling and, ultimately, to a phase transition from atomic to molecular gas Morris (2006). Therefore the molecular gas that defines the loops is constantly replenished. This scenario also confirms our results, because the gas in the foot point of the loop has not yet been processed by star formation.

\subsection{Gas accretion from the outer disk}

We have also found high isotopic ratios toward the noncircular components in the nuclear disk, which have been explained in terms of a stellar-bar driven potential (Binney et al. 1991). The 1.3 complex has been observed and studied by several authors (Tanaka et al. 2007; Oka et al. 2001; Hüttemeister et al. 1998). This molecular complex shows broad velocity widths and a large latitudinal height scale. The molecular emission shows two velocity components, one centered at $\sim 110 \mathrm{~km} \mathrm{~s}^{-1}$ and the other at $\sim 190 \mathrm{~km} \mathrm{~s}^{-1}$, which correspond to our Disk X2-1 and Disk X1-1 sources, respectively. Tanaka et al. (2007) find an enhancement of the CO $J=3-2, \mathrm{HCO}^{+} J=1-0, \mathrm{HCN} J=1-0$ with respect to the $\mathrm{CO} J=1-0$, and an enhancement in the abundance of $\mathrm{SiO}$ in the high-velocity component $\left(>110 \mathrm{~km} \mathrm{~s}^{-1}\right)$. They also identify several expanding shells that are very prominent at $v_{\text {LSR }}<110 \mathrm{~km} \mathrm{~s}^{-1}$ associated with recent star formation. They conclude that the two components correspond to different types of gas with different kinematics and physical conditions. Our Disk X1-1 component presents an enhancement of the $\mathrm{SiO} /{ }^{13} \mathrm{CO}$ intensity ratio of a factor $\sim 3$ (Tanaka et al. 2007) with respect to the lower velocity component (Disk X2-1). Like Hüttemeister et al. (1998), Tanaka et al. (2007) explains the high SiO abundance as a consequence of recent shock activity.

From our measurements of isotopic ratios, we conclude that the gas associated with the lowest velocity component presents a "typical" GC isotopic ratio consistent with previous ideas of chemistry dominated by shocks generated by supernova explosions. It is very unlikely, however, that the higher velocity component, Disk X1-1, could be associated to the supernova scenario, like the Disk X2-1 component does, as also inferred from the high isotopic ratio found toward this source. The most likely scenario is that the high $\mathrm{SiO}$ abundance is the result of the shocks generated by the transfer of gas between the X1 and X2 orbits as suggested by the potential bar scenario.

\section{Conclusions}

We have determined lower limits to the ${ }^{12} \mathrm{C} /{ }^{13} \mathrm{C}$ isotopic ratio toward 9 positions in the $\mathrm{GC}$ region, both in the disk and in the halo. In contrast to the values found in the disk sources $(\sim 4$ 30 ), we found high isotopic ratios toward the locations where the disk-halo $(\sim>40->70)$ and X1-X2 orbit interactions ( $>42-$ $>56$ ). Our results are consistent with a scenario where gas from the halo is accreted to the disk and with the transfer of gas from the outskirt of the disk to the GC through X1 and X2 orbits as suggested by the potential bar scenario.

Acknowledgements. D.R. and R.M. were supported by DGI grant AYA 2008-06181-C02-02. J.M-P. and S.M. have been partially supported by the Spanish 74 MICINN under grant number ESP2007-65812-C02-01, and by AYA2010-21697-C05-01. L.B. acknowledges support from FONDAP Center for Astrophysics 15010003 and from Center of Excellence in Astrophysics and Associated Technologies (PFB 06).

\section{References}

Audouze, J. 1985, in ESO Astroph. Symp., ed. I. J. Danziger, F. Matteucci, \& K. Kjar, 21, 373

Bally, J., \& Langer, W. D. 1982, ApJ, 255, 143

Binney, J., Gerhard, O. E., Stark, A. A., Bally, J., \& Uchida, K. I. 1991, MNRAS, 252, 210

Chu, Y., \& Watson, W. D. 1983, ApJ, 267, 151

Contopoulos, G., \& Papayannopoulos, T. 1980, A\&A, 92, 33

Fuente, A., Rodríguez-Franco, A., García-Burillo, S., Martín-Pintado, J., \& Black, J. H. 2003, A\&A, 406, 899

Fukui, Y., Yamamoto, H., Fujishita, M., et al. 2006, Sci., 314, 106

García-Burillo, S., Combes, F., Schinnerer, E., Boone, F., \& Hunt, L. K. 2005, A\&A, 441, 1011

Ghez, A. M., Salim, S., Hornstein, S. D., et al. 2005, ApJ, 620, 744

Glassgold, A. E., Huggins, P. J., \& Langer, W. D. 1985, ApJ, 290, 615

Graedel, T. E., Langer, W. D., \& Frerking, M. A. 1982, ApJS, 48, 321

Henkel, C., Güsten, R., \& Gardner, F. F. 1985, A\&A, 143, 148

Horiuchi, T., Matsumoto, R., Hanawa, T., \& Shibata, K. 1988, PASJ, 40, 147

Hüttemeister, S., Dahmen, G., Mauersberger, R., et al. 1998, A\&A, 334, 646

Langer, W. D., Graedel, T. E., Frerking, M. A., \& Armentrout, P. B. 1984, ApJ, 277,581

Langer, W. D., \& Penzias, A. A. 1990, ApJ, 357, 477

Langer, W. D., Wilson, R. W., Henry, P. S., \& Guélin, M. 1978, ApJ, 225, L139

Machida, M., Matsumoto, R., Nozawa, S., et al. 2009, PASJ, 61, 411

Maciejewski, W., \& Sparke, L. S. 2000, MNRAS, 313, 745

Martín, S., Requena-Torres, M. A., Martín-Pintado, J., \& Mauersberger, R. 2008, ApJ, 678, 245

Matsumoto, R., Horiuchi, T., Shibata, K., \& Hanawa, T. 1988, PASJ, 40, 171

Mauersberger, R., \& Henkel, C. 1993, Rev. Modern Astron., 6, 69

Meyer, B. S. 1994, ARA\&A, 32, 153

Milam, S. N., Savage, C., Brewster, M. A., Ziurys, L. M., \& Wyckoff, S. 2005, ApJ, 634, 1126

Morris, M. R. 2006, Sci., 314, 70

Morris, M., \& Serabyn, E. 1996, ARA\&A, 34, 645

Morris, M., Ghez, A. M., \& Becklin, E. E. 1999, Adv. in Space Res., 23, 959

Oka, T., Hasegawa, T., Sato, F., et al. 2001, ApJ, 562, 348

Prantzos, N., Aubert, O., \& Audouze, J. 1996, A\&A, 309, 760

Riquelme, D., Bronfman, L., Mauersberger, R., May, J., \& Wilson, L. T. 2010, 115, A\&A, accepted [arXiv: 1006.3438]

Rodríguez-Fernández, N. J., Martín-Pintado, J., de Vicente, P., \& Fuente, A. 2002, Ap\&SS, 281, 331

Stark, A. A. 1981, ApJ, 245, 99

Stark, A. A., Martin, C. L., Walsh, W. M., et al. 2004, ApJ, 614, L41

Takahashi, K., Nozawa, S., Matsumoto, R., et al. 2009, PASJ, 61, 957

Tanaka, K., Kamegai, K., Nagai, M., \& Oka, T. 2007, PASJ, 59, 323

Torii, K., Kudo, N., Fujishita, M., et al. 2009, PASJ, 62, 1307

Torii, K., Kudo, N., Fujishita, M., et al. 2010, PASJ, 62, 675

Usero, A., García-Burillo, S., Fuente, A., Martín-Pintado, J., \& RodríguezFernández, N. J. 2004, A\&A, 419, 897

Wannier, P. G. 1980, ARA\&A, 18, 399

Wilson, T. L. 1999, Rep. Progr. Phys., 62, 143

Wilson, T. L., \& Matteucci, F. 1992, A\&A Rev., 4, 1

Wilson, T. L., Rohlfs, K., \& Hüttemeister, S. 2009, Tools of Radio Astronomy (Springer-Verlag)

Woods, P. M., \& Willacy, K. 2009, ApJ, 693, 1360 